\title{
PROJETO DO CURSO DE ENGENHARIA CIVIL DA UNIVERSIDADE POSITIVO COM FOCO NAS COMPETÊNCIAS DO EGRESSO
}

DOI: 10.37702/2175-957X.COBENGE.2021.3456

\author{
Patrícia Lizi de Oliveira Maggi - plomaggi@gmail.com \\ Universidade Positivo \\ Domingos Benatto 72 \\ 82320-590 - Curitiba - PR
}

Carlos Alberto de Moraes Vasconcellos - cav@up.edu.br

Universidade Positivo

Rua Francisco Parise 116

82015-090 - Curitiba - PR

Resumo: Este texto apresenta a experiência do curso de Engenharia Civil da Universidade Positivo na implantação de uma matriz curricular com foco em competências. A reformulação da matriz foi motivada pela então Minuta das novas Diretrizes Curriculares Nacionais e pelo diagnóstico realizado junto a setores da sociedade que apontavam lacunas na formação do engenheiro em relação às demandas do mercado de trabalho. A nova concepção de curso, que daria origem à nova matriz curricular, deveria dar maior ênfase às habilidades do egresso e desenvolver atitudes necessárias para a atuação profissional, sem negligenciar a importância do conteúdo. O trabalho, desenvolvido pelo Núcleo docente estruturante do curso, foi realizado em sincronia com o projeto dos demais cursos de engenharia da Universidade. Iniciou-se pelo mapeamento das competências do egresso, seguido da escolha de atividades educacionais obrigatórias para então ter início o desenho da matriz e de suas componentes curriculares. Ao contrário do modelo tradicional, onde priorizam-se atividades cognitivas menos complexas (lembrar, entender) nas séries iniciais, para, depois, introduzir atividades de aplicação, análise até chegar em criação, adotou-se um modelo no qual todas as componentes curriculares teriam objetivos de aprendizagem de dimensão cognitiva mais elevada (a partir de 3 na taxonomia de Bloom) e o escalonamento do aprendizado se daria pela complexidade das tarefas que o aluno deveria desempenhar. Portanto, mesmo disciplinas da primeira série já têm objetivos de dimensões mais elevadas, como, por exemplo, desenvolver projeto. Ao longo do curso aumenta a complexidade dos trabalhos requeridos. Desta forma, disciplinas clássicas deixaram de configurar na matriz e seus conteúdos foram distribuídos 
entre várias componentes curriculares que demandam seu conhecimento para a realização das atividades propostas. Esta nova matriz foi implantada no $1^{\text {o }}$ semestre de 2019 e, portanto, podem ser apresentados alguns resultados parciais. Apesar do receio do corpo docente de que poderiam receber avaliações de desempenho piores, por parte dos alunos, isso não se verificou. A média da avaliação dos professores manteve-se, praticamente inalterada. Os estudantes declaram interesse em desenvolver as atividades propostas, mas reclamam do volume de trabalho e do tempo de dedicação exigido. Os professores observam o interesse dos alunos, mas apontam maior dificuldade na preparação das aulas e demanda de tempo para acompanhamento dos estudantes.

Palavras-chave: Projeto de curso, Matriz curricular por competências, Engenharia Civil 


\section{INTRODUÇÃO}

Em abril de 2019 o Ministério da Educação publicou a resolução n 2/2019 que institui as novas Diretrizes Curriculares Nacionais de Engenharia. Antes disso, a minuta do texto foi disponibilizada para análise da comunidade acadêmica. $O$ texto deixa clara a intenção de que os cursos de engenharia passem a focar o ensino nas competências do egresso, em complementação ao modelo tradicional que foca no conteúdo a ser aprendido.

Tendo analisado essa proposta e compreendido que se tratava de um avanço significativo no ensino de engenharia, a Universidade Positivo tomou a decisão de mudar seus projetos de curso incorporando as premissas do ensino com foco em competências e implantar suas novas matrizes curriculares, no $1^{\circ}$ semestre letivo de 2019 . Para tanto, a concepção das matrizes curriculares a partir dessa nova visão foi desenvolvida ao longo do $2^{\circ}$ semestre de 2018. Maggi (2020) apresenta o processo colaborativo adotado para a construção das matrizes de todos os cursos de engenharias da IES.

Este texto apresenta o trabalho específico do curso de Engenharia Civil e traz alguns resultados parciais medidos ao longo de dois anos, a partir do início da implantação.

Ao final de 2020, foi aprovado o novo Plano de Desenvolvimento Institucional da UP com o propósito de se fazer Educação para o Desenvolvimento Sustentável. As matrizes dos cursos foram adequadas à essa nova diretriz e implantadas em 2021. No caso da Engenharia Civil houveram algumas pequenas mudanças em relação ao projeto de 2019.

\section{COMPETÊNCIAS DO EGRESSO}

Antes de iniciar o projeto deste novo modelo de curso, foi necessário entender o significado de se fazer ensino por competências. Bloom (1976) divide os objetivos de aprendizagem em:

- Objetivos globais

- Objetivos específicos

- Objetivos instrucionais

Conhecida essa divisão, o grupo de trabalho, decidiu que, para se fazer ensino por competência, os objetivos globais do curso deveriam ser expressos na forma de competências do egresso, entendendo-se a definição de competência a partir de suas três componentes: conhecimento (saber), habilidade (saber-fazer) e atitude (saber ser / saber agir). Os objetivos específicos das componentes curriculares deveriam ser selecionados de forma a construir as competências globais e os objetivos instrucionais das atividades de ensino deveriam proporcionar o aprendizado que levassem aos objetivos específicos.

Desta forma, a definição do novo projeto de curso começou pelo mapeamento dos objetivos globais, ou seja, das competências do egresso que levam em conta:

- O PDI

- As novas diretrizes curriculares nacionais

- As demandas da sociedade, pesquisadas por meio de encontros entre o NDE do curso e representantes de diferentes setores

- Os requisitos do CREA-PR para habilitação profissional 
O trabalho de redação dessas competências foi interativo e os textos foram sofrendo modificações ao longo do processo, desde o mapeamento inicial até o detalhamento dos planos de ensino das componentes curriculares. Listam-se as competências do egresso do curso de Engenharia Civil, conforme se encontram hoje:

1. Analisar e compreender os fenômenos físicos e químicos por meio de modelos, verificados e validados por experimentação.

2. Atuar na engenharia com ética, pensamento crítico e protagonismo na solução dos problemas da sociedade, conhecendo e aplicando a legislação e os atos normativos no âmbito do exercício da profissão.

3. Conceber, planejar, coordenar e executar projetos de engenharia com atitude inovadora e empreendedora, atendendo às necessidades dos usuários, de acordo com os valores de uma sociedade sustentável.

4. Agir com responsabilidade social, visão crítica e pensamento sistêmico, atuando como agente transformador na sociedade.

5. Atuar no mundo do trabalho mobilizando e desenvolvendo conhecimento científico de forma coletiva em prol da solução de problemas ambientais.

6. Desenvolver soluções inovadoras para problemas da sociedade, mobilizando conhecimento científico, com visão holística e humanista.

7. Promover desenvolvimento econômico e social com atitude empreendedora, mobilizando conhecimento técnico e científico, com visão holística e humanista e com atenção à necessidade de preservação do meio ambiente.

8. Comunicar-se, efetivamente, com empatia, nas formas escrita, oral e gráfica.

9. Trabalhar em equipes multidisciplinares de forma cooperativa, empática, resiliente e responsiva, considerando as singularidades e diferenças socioculturais dos indivíduos.

10. Aprender de forma autônoma e lidar com situações e contextos complexos, buscando constante atualização e atuando na disseminação dos avanços da ciência e da tecnologia com atitude resiliente frente aos desafios de inovação.

11. Elaborar estudos geológicos e geotécnicos visando aplicação em obras de engenharia.

12. Projetar obras geotécnicas considerando critérios de segurança, otimizando recursos e minimizando impactos ambientais.

13. Elaborar estudos hidrológicos visando a garantia da sustentabilidade dos recursos hídricos.

14. Projetar obras hidráulicas e de saneamento considerando aspectos econômicos, sociais e ambientais.

15. Projetar sistemas para gestão de resíduos sólidos considerando aspectos econômicos, sociais e ambientais.

16. Planejar sistemas de transporte promovendo desenvolvimento socioeconômico e minimizando os impactos das interferências humanas sobre o meio ambiente.

17. Projetar elementos de infraestrutura de transportes seguindo critérios de segurança, buscando soluções sustentáveis, inovadoras e que atendam às necessidades dos usuários.

18. Projetar soluções arquitetônicas criativas de modo a atender às necessidades humanas relacionadas à segurança, conforto e acessibilidade, considerando aspectos técnicos, sociais e ambientais.

19. Planejar, implementar, supervisionar e controlar obras civis analisando e compreendendo as necessidades do usuário e seu contexto, seguindo critérios de segurança, conforto, economia e sustentabilidade ambiental. 
20. Desenvolver concretos para uso na construção civil visando racionalização dos recursos e consequente redução dos impactos ambientais.

21. Projetar sistemas de instalações prediais seguindo critérios de segurança, buscando soluções sustentáveis e que atendam às necessidades dos usuários.

22. Desenvolver estudos preliminares de projetos de barragens e reservatórios ponderando os impactos sociais, econômicos e ambientais.

23. Projetar estruturas seguindo critérios de segurança, buscando soluções sustentáveis e que atendam às necessidades dos usuários.

\section{ESCOLHA E DISTRIBUIÇÃO DAS COMPONENTES CURRICULARES}

A partir da definição das competências do egresso, foram escolhidas atividades educacionais que deveriam ser vivenciadas pelos estudantes a fim de desenvolver essas competências. Para facilitar a escolha dessas atividades, definiram-se, primeiramente, cinco linhas de atuação:

- Modelagem e programação (comum a todas as engenharias)

- Construção civil e materiais

- Estruturas

- Geotecnia e Transportes

- Hidráulica, Saneamento e Meio ambiente

Com o novo PDI (2021-2024) acrescentou-se a sexta linha: Pilares do Desenvolvimento Sustentável.

\subsection{Classificação dos objetivos de aprendizagem}

O conjunto de atividades de cada linha foi definido de tal forma a garantir que a dimensão das competências a ele associadas fosse atingida. Para tanto, as competências foram classificadas de acordo com uma associação entre as dimensões cognitivas da taxonomia de Bloom revisada (Quadro 1) e a Taxonomia de Fink (Figura 1).

Quadro 1 - Classificação de habilidades de acordo com a

Taxonomia de Bloom revisada, adaptado de Revised

Bloom's taxonomy (2019)

\begin{tabular}{|c|c|c|c|c|c|}
\hline \multirow{2}{*}{\multicolumn{2}{|c|}{$\begin{array}{l}\text { Os verbos representam objetivos de } \\
\text { aprendizagem, não atividades de } \\
\text { aprendizagem. Pensar da seguinte } \\
\text { forma: Estudantes serão capazes de .. }\end{array}$}} & \multicolumn{4}{|c|}{ Dimensões do conhecimento } \\
\hline & & Factual & Conceitual & Procedimental & Metacognitivo \\
\hline \multirow{6}{*}{ 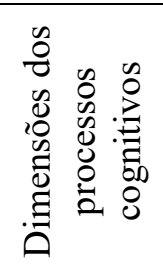 } & Lembrar & Listar & Reconhecer & Recordar & Identificar \\
\hline & Entender & Resumir & Classificar & Esclarecer & Prever \\
\hline & Aplicar & Responder & Providenciar & Executar & Usar \\
\hline & Analisar & Selecionar & Diferenciar & Integrar & Desconstruir \\
\hline & Avaliar & Selecionar & Determinar & Julgar & Refletir \\
\hline & Criar & Generalizar & Montar & Projetar & Criar \\
\hline
\end{tabular}


Figura 1 - Taxonomia de Fink, adaptado de O’Neill \& Murphy (2010)



De acordo com a característica predominante, cada objetivo global, foi classificado com um número ou com uma letra, conforme indicado no quadro 2.

Quadro 2 - Combinação das taxonomias de Bloom e de

Fink, conforme adotado pela UP, para classificação das competências

\begin{tabular}{|c|c|c|c|c|c|c|c|c|c|}
\hline \multicolumn{10}{|c|}{ Taxonomia de Fink } \\
\hline Conhecim & to básico & \multicolumn{4}{|c|}{ Aplicação } & \multirow{3}{*}{$\begin{array}{c}\text { I. } \\
\text { Integradora }\end{array}$} & \multirow{3}{*}{$\begin{array}{c}\mathrm{H} . \\
\text { Humana }\end{array}$} & \multirow{3}{*}{$\begin{array}{c}\text { C. } \\
\text { Cuidado }\end{array}$} & \multirow{3}{*}{ L. } \\
\hline \multicolumn{6}{|c|}{ Taxonomia de Bloom revisada } & & & & \\
\hline 1. Lembrar & 2. Entender & 3. Aplicar & 4. Analisar & 5. Avaliar & 6. Criar & & & & \\
\hline
\end{tabular}

\subsection{Forma de progressão ao longo do curso}

A progressão do aprendizado ao longo do curso pode se dar de diferentes formas. A mais tradicional constitui-se na escalada das dimensões cognitivas iniciando pelos aprendizados mais básicos (dimensões 1 e 2 de Bloom) para, depois, atingir as dimensões mais elevadas.

Ao identificar que todas as competências do egresso tinham dimensões cognitivas acima de 3, o NDE do curso escolheu fazer a escalada do aprendizado aumentando gradativamente a complexidade dos problemas aos quais o aluno seria confrontado, mas iniciando, já nas primeiras séries, com atividades compatíveis com as dimensões cognitivas mais elevadas.

Desta forma, a principal estratégia de aprendizado adotada foi a metodologia de projetos, apesar de não ser a única. Assim, foram elaboradas atividades educacionais que priorizam fazer e tomar decisões. Os conhecimentos básicos vão sendo adquiridos ao passo que os problemas, e principalmente os projetos, se apresentam, pois, o aluno precisa buscar esses conhecimentos para conseguir resolver os desafios.

\subsection{Distribuição das atividades educacionais em componentes curriculares}

Depois de definido, em linhas gerais, quais atividades os estudantes precisariam vivenciar ao longo do curso, essas atividades foram distribuídas em componentes curriculares, levando em conta o equilíbrio entre as linhas de atuação, a carga horária 
necessária para cada atividade e o grau de amadurecimento do estudante em cada etapa do curso.

Com essa forma de distribuição, a matriz curricular desenvolvida não apresenta as disciplinas clássicas das engenharias, que priorizam a divisão por conteúdo. O conteúdo está distribuído e agrupado nas várias componentes, conforme a demanda da atividade prevista. Desta forma, obteve-se uma matriz mais interdisciplinar buscando desenvolver uma visão sistêmica dos problemas de engenharia.

Citam-se como exemplo as disciplinas de Modelagem matemática I a IV que agrupam os conhecimentos de Cálculo, Física, Álgebra, Geometria Analítica, Cálculo Numérico e Química, focando na habilidade de modelagem do estudante, ou seja, na aplicação dessas ferramentas na modelagem de problemas reais.

A linha de Construção Civil e Materiais, apresentada aqui como exemplo, é constituída das componentes indicadas no quadro 3 , onde são apresentadas também as atividades educacionais de cada uma delas.

Quadro 3 - Componentes curriculares e atividades da linha de Construção Civil e Materiais

\begin{tabular}{|c|c|c|}
\hline Componente & Período & Atividades \\
\hline $\begin{array}{c}\text { Desenho técnico e de } \\
\text { construção }\end{array}$ & $1^{\circ}$ & $\begin{array}{c}\text { Desenho, em software de Desenho (AutoCad), } \\
\text { de uma residência unifamiliar, representando } \\
\text { plantas, cortes e elevações. }\end{array}$ \\
\hline $\begin{array}{c}\text { Planejamento e } \\
\text { gerenciamento de obras I }\end{array}$ & $1^{\circ}$ & $\begin{array}{c}\text { Memorial descritivo, orçamento por meio de } \\
\text { custos unitários e planejamento por diagrama de } \\
\text { Gantt de uma obra de construção de residência } \\
\text { unifamiliar. }\end{array}$ \\
\hline $\begin{array}{c}\text { Modelagem da informação } \\
\text { na construção }\end{array}$ & $2^{\circ}$ & $\begin{array}{c}\text { Soluções de problemas usando ferramentas BIM } \\
\text { até 4D. }\end{array}$ \\
\hline $\begin{array}{c}\text { Tecnologia do concreto } \\
\text { gerenciamento de obras II }\end{array}$ & $3^{\circ}$ & $\begin{array}{c}\text { Dosagens de concretos por métodos } \\
\text { experimentais. }\end{array}$ \\
\hline $\begin{array}{c}\text { Planejamento e } \\
\text { gerenciamento de obras } \\
\text { III }\end{array}$ & $4^{\circ}$ & $\begin{array}{c}\text { Especificações técnicas, orçamento e o } \\
\text { planejamento de uma obra de construção de } \\
\text { edifício de múltiplos pavimentos. }\end{array}$ \\
\hline $\begin{array}{c}\text { Projeto de instalações } \\
\text { hidráulicas prediais }\end{array}$ & $5^{\circ}$ & $\begin{array}{c}\text { Projeto de canteiro, especificações técnicas, } \\
\text { orçamento e o planejamento de uma obra de } \\
\text { infraestrutura. }\end{array}$ \\
\hline $\begin{array}{c}\text { Projeto de instalações } \\
\text { elétricas prediais }\end{array}$ & $7^{\circ}$ & $\begin{array}{c}\text { Projeto de instalações elétricas de uma } \\
\text { edificação de pequeno porte. }\end{array}$ \\
\hline $\begin{array}{c}\text { Projeto de instalações hidráulicas de uma } \\
\text { edificação de pequeno porte. }\end{array}$ \\
\hline
\end{tabular}

Um outro exemplo é a linha de Estruturas, dividida em 8 componentes curriculares: Projeto de estruturas I a VIII. Na primeira o estudante identifica sistemas e características dos materiais estruturais. A partir da segunda ele começa a desenvolver projetos de estruturas, partindo do projeto de uma estrutura treliçada de madeira, passando por pórtico de aço até chegar num edifício de concreto armado. Os conteúdos de Estática, Resistência dos materiais e Teoria das estruturas são estudados conforme vão sendo necessários nos projetos em desenvolvimento. Ao final do percurso, o aluno deverá ter aprendido os conceitos básicos que eram antes estudados nas disciplinas iniciais, mas eles são introduzidos, de forma aplicada, ao longo do trajeto. 
Com modelo semelhante foram desenhadas as demais linhas do curso, conforme ilustrado na figura 2.

Figura 2 - Representação esquemática do modelo de progressão ao longo de uma linha de atuação do curso

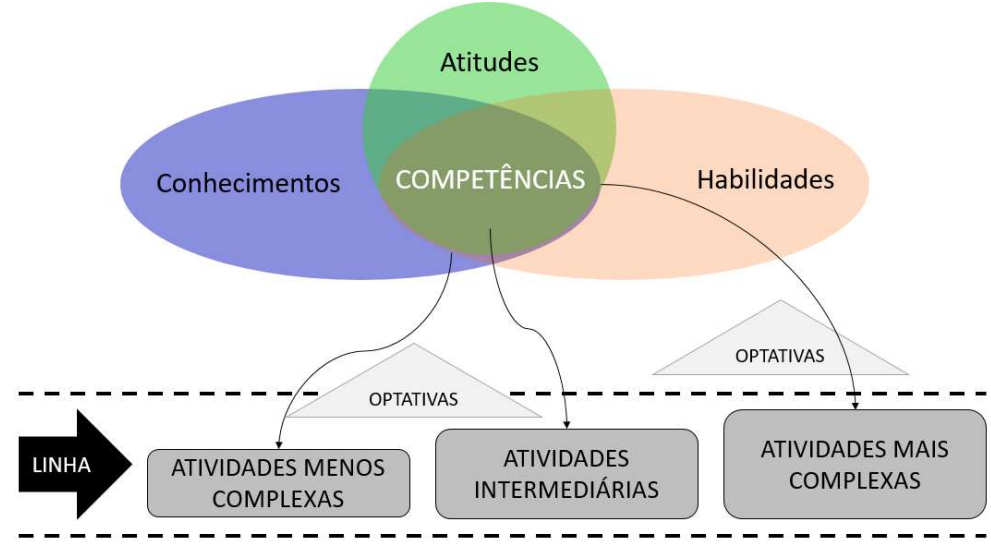

\subsection{Habilidades comportamentais e atitudes}

Durante o mapeamento das competências do egresso o "projetar" destacou-se entre as habilidades técnicas. Entretanto, algumas habilidades comportamentais apareceram com bastante importância, tais como: comunicação, criatividades e empreendedorismo; além de atitudes como empatia, responsabilidade, responsividade e ética.

Essas habilidades podem e devem ser trabalhadas ao longo do curso, em paralelo às habilidades técnicas, mas percebeu-se a importância de se dar uma atenção especial a algumas delas, a fim de garantir um desenvolvimento mais aprofundado. Na matriz original, de 2019, foram, então, inseridas as componentes curriculares: LCl (Laboratório de criatividade e inovação) I a X. Essas componentes eram comuns a todos os cursos da área de exatas (Engenharias, Tecnologia da Informação e Arquitetura) e os alunos eram matriculados em turmas mistas. Para as duas primeiras os alunos recebiam o desafio de identificar e apresentar uma solução para uma questão social. Na terceira e na quarta eles deveriam apresentar uma solução inovadora a um problema. Na quinta e na sexta desenvolveriam um plano de negócio. Na sétima e na oitava desenvolveriam atividades relacionadas ao comportamento corporativo e nas duas últimas trabalhariam as habilidades de comunicação.

$\mathrm{Na}$ revisão da matriz, os $\mathrm{LCl}$ foram substituídos por:

- Pesquisa e sociedade

- Pesquisa aplicada e meio ambiente

- Projeto de inovação

- Projeto empreendedor

- Comunicação assertiva

As quatro primeiras fazem parte da extensão institucional. São disciplinas comuns a todos os cursos da Universidade. As turmas recebem alunos de todas as escolas, a citar:

- Escola de Medicina

- Escola de Ciências da Saúde

- Escola Politécnica

- Escola de Negócios

- Escola de comunicação de design

- Escola de Direito e Ciências sociais

- Escola de Tecnologia da Informação 

multidisciplinares.

Comunicação assertiva é comum a todas as Engenharias e nela os estudantes irão trabalhar as habilidades de comunicação e feedback, usando o Trabalho de Conclusão de Curso como objeto para provocar as discussões.

\section{RESULTADOS OBTIDOS NOS DOIS PRIMEIROS ANOS DE IMPLANTAÇÃO}

Com dois anos de implantação deste projeto de curso, apresentam-se alguns resultados parciais.

\subsection{Impacto na avaliação individual dos professores}

Ao conhecerem a proposta do novo projeto de curso das engenharias, um grupo significativo de professores demostrou preocupação com relação à avaliação docente. $A$ ADOC é um dos instrumentos aplicados pela CPA e que mede a impressão dos alunos em relação à atuação dos professores. $O$ estudante responde à 4 perguntas, atribuindo uma nota de 1 a 10 para cada questão:

- Como você avalia o domínio do conteúdo?

- Como você avalia o relacionamento do professor com alunos em sala de aula?

- Como você avalia a coerência entre as avaliações e o conteúdo abordado na disciplina?

- Como você avalia a organização e didática?

O receio dos professores era de que perderia pontos no item organização e didática ao adotarem a metodologia de projetos em substituição às aulas expositivas, com as quais os alunos foram acostumados ao longo de sua trajetória educacional.

Comparando-se a ADOC média dos professores que atuaram na $1^{\text {a }}$ série do curso em 2019 com seus resultados de 2018, observou-se uma variação de 8,71 para 8,86. Podese concluir, portanto, que a implantação das atividades de aprendizado propostas pelo novo projeto de curso não teve impacto negativo sobre a avaliação do desempenho dos professores.

\subsection{Impressões dos professores após a implantação da nova matriz}

Apresentam-se a seguir pontos positivos e dificuldades identificadas pelos professores que atuaram na nova matriz do curso em 2019. Excluíram-se as impressões sobre o ano letivo de 2020 que foi gravemente impactado pela pandemia, durante a qual a Universidade substituiu o ensino presencial pelo ensino remoto emergencial.

Pontos positivos:

- Os alunos demonstram interesse em resolver problemas práticos, do mundo real;

- Alunos gostaram de enxergar os projetos como um todo, tendo a visão global do problema;

- Ao desenvolver um projeto, o aluno enxerga o produto, já despertando uma visão mais empreendedora;

- Disciplinas específicas do curso têm aderência natural ao modelo de desenvolvimento de competências;

- As habilidades de dimensões cognitivas mais altas tais como modelar, analisar, projetar, formam o profissional de forma mais completa.

Desafios a serem superados: 
- As dimensões cognitivas do tipo modelar, analisar, projetar, requerem domínio das dimensões inferiores, do tipo conhecer e aplicar, e o professor precisa encontrar meios do aluno se apropriar desses conhecimentos;

- Há necessidade de sincronia temporal entre disciplinas de formação profissional e núcleo de modelagem (comum às engenharias), para que o aluno tenha desenvolvido as habilidades necessárias para os projetos;

- Disciplinas do núcleo comum, em especial Modelagem Matemática, demandam professores com formação multidisciplinar;

- Há falta de referências bibliográficas orientadas ao desenvolvimento de competências de dimensões elevadas. Em sala de aula o aluno é estimulado a modelar e projetar, a partir de situações elaboradas pelo professor, mas na bibliografia encontram-se exercícios de memorização, entendimento e aplicação;

- Disciplinas de projeto não têm solução única, logo o acompanhamento e a avaliação demandam mais tempo do professor;

- Como houve mudança na distribuição dos conteúdos e na forma de avaliação, é difícil estabelecer uma métrica para comparar o aprendizado no modelo antigo e no modelo novo, antes de se concluir um ciclo completo.

\subsection{Impressões dos alunos após a implantação da nova matriz}

Apresentam-se a seguir um resumo de pontos positivos e dificuldades identificadas pelos alunos.

Pontos positivos:

- A nova matriz é motivadora por tratar assuntos de engenharia de forma aplicada desde o primeiro período do curso;

- A nova metodologia instiga o pensamento lógico e a busca de conhecimento;

- É possível relacionar competências desenvolvidas nas disciplinas com as atividades vivenciadas no estágio;

- Além de competências técnicas, estão sendo trabalhadas as competências comportamentais;

- A avaliação por projeto é mais justa que a avaliação por prova;

- A avaliação contínua estimula o estudo constante;

- O estudante se sente mais preparado para o mercado de trabalho.

Desafios:

- Os professores precisam garantir o desenvolvimento dos objetivos de dimensão cognitiva mais elevada e os conhecimentos básicos fundamentais;

- É mais trabalhoso e demanda mais tempo de dedicação do aluno;

- A Universidade precisa elaborar um calendário de forma a não acumular as entregas de projetos;

- Encontrar problemas de engenharia que interessem a todos os públicos nas disciplinas do núcleo comum;

- É preciso quebrar paradigmas na forma de estudar. Não existe gabarito com solução única.

\section{CONSIDERAÇÕES FINAIS}

Este artigo apresenta a experiência na implantação de um projeto de curso com foco nas competências do egresso e as escolhas feitas pelo curso de Engenharia Civil da Universidade Positivo para a sequência de atividades a serem desenvolvidas no percurso do aluno. Nesta proposta, o aluno teria contato com problemas práticos desde as primeiras 
séries do curso. Para tanto, foram selecionadas atividades, para as diversas linhas de atuação, de tal forma que habilidades complexas fossem trabalhadas desde o início e a dificuldade dos problemas a serem resolvidos aumentasse ao longo do tempo, demandando novos conhecimentos. As componentes curriculares não foram divididas com base nos conteúdos, mas nessas atividades de aprendizagem. Portanto, os conteúdos não aparecem mais nas disciplinas clássicas que são comumente encontradas nos cursos de engenharia, mas foram distribuídos em várias disciplinas práticas, a maioria delas como foco em projeto. As competências comportamentais são desenvolvidas tanto nas disciplinas técnicas quanto em disciplinas especificamente desenhadas para este fim.

Durante o desenvolvimento da proposta, assim como nos primeiros meses de implantação, um grupo significativo de professores demonstrou receio de ter queda nas avaliações de seu desempenho em função de uma impressão negativa dos estudantes, o que não se verificou. A média nas avaliações do desempenho dos professores se manteve praticamente inalterada, apresentando uma leve elevação que não pode ser atribuída ao novo projeto de curso.

Foi necessário um treinamento específico para a implantação das atividades propostas e os professores precisaram ser tranquilizados com relação às cobranças da Universidade, para que aderissem à nova proposta. Depois de dois anos, a resistência do grupo de professores diminuiu, mas não desapareceu. O grupo pôde observar a boa adesão por parte dos alunos e um incremento no grau de complexidade dos aprendizados, mas ainda tem algumas dúvidas com relação aos resultados finais. Apesar de boas impressões, a medida definitiva do aprendizado só será possível ao final de um ciclo completo.

\section{REFERÊNCIAS}

ANDERSON, L. W.; KRATHWOHL, D. R. (Eds.) A Taxonomy for Learning, Teaching, and Assessing: a revision of Bloom's Taxonomy of Educational Objectives. New York: Longman, 2001.

BLOOM, B. S. et al. Taxonomia de objetivos educacionais: Domínio Cognitivo. Porto Alegre: Editora Globo, 1976.

BRASIL. Conselho Nacional de Educação. Resolução CNE/CES n.2/2019. Disponível em:

http://portal.mec.gov.br/index.php?option=com_docman\&view=download\&alias=112681rces002-19\&category_slug=abril-2019-pdf\&Itemid=30192. Acesso em: 30 set. 2019.

MAGGI, Patrícia L. O. (2000). Construção de matrizes por competência: a experiência das engenharias da Universidade Positivo. In: XLVIII Congresso Brasileiro de Educação em Engenharia, 2020, Bento Gonçalves. Anais. Bento Gonçalves.

O’NEIL, G.; MURPHY, F. (2010). Guide to Toxonomies of Learning. Disponível em https://www.ucd.ie/t4cms/ucdtla0034.pdf, Acesso em: 14 abril 2021.

REVISED Bloom's taxonomy. Iowa State University, 2018. Disponível em https://www.celt.iastate.edu/teaching/effective-teaching-practices/revised-bloomstaxonomy/. Acesso em: 15 ago 2018. 


\title{
CIVIL ENGINEERING UNDER GRADUATION PROJECT FOCUSING ON EGRESS SKILLS: THE EXPERIENCE OF POSITIVO UNIVERSITY
}

\begin{abstract}
This document presents the experience of the Civil Engineering under graduation at Universidade Positivo in the implementation of a curricular matrix focused on competences (outcomes). The reformulation of the curriculum was motivated by the release of the new National Curriculum Guidelines and the diagnosis carried out with sectors of society that pointed out gaps in the formation of the engineer in relation to the demands of the profession. The new under graduation design, which give rise to the new curriculum, should place greater emphasis on the student's skills and develop the necessary attitudes for professional performance, without neglecting the importance of the content. Developed by the teaching staff, the work was carried out in synchrony with the design of the other engineering under graduation programs at the University. It started with the mapping of the graduates' competences, followed by the choice of mandatory educational activities and the design of the curriculum and its components. In the traditional model, less complex cognitive activities (remember, understand) are prioritized in the initial periods. Only after that, practical activities, analysis and projects are introduced. In the new adopted model all the curriculum components have learning objectives of higher cognitive dimension (from 3 to 6 in Bloom's taxonomy) and the staggering of learning would be due to the complexity of the tasks that the student should perform. Therefore, even courses in the first grade already have higher dimension objectives, such as, for example, developing a project. Throughout the program the complexity of the required jobs increases. In this way, classic disciplines are no longer present in the curriculum and their contents were distributed among several curricular components that require their knowledge to carry out the proposed activities. This new curriculum was implemented in the 1st semester of 2019 and, therefore, some partial results can be presented. Despite the fear of the faculty, the average of the teachers' performance evaluation remained practically unchanged. Students declare an interest in developing the proposed activities, but complain about the amount of work. The teachers observe the students' interest, but they point out greater difficulty in preparing the classes and demand time to monitor the students.
\end{abstract}

Keywords: graduation design, curriculum, Civil Engineering, student skills 